\title{
Representation of Harmonic Functions in the Lie Ball by Dirichlet Series
}

\author{
Mitsuo MORIMOTO and LÊ Hai Khôi* \\ Sophia University and Hanoi Institute of Information Technology
}

\begin{abstract}
We prove that complex harmonic functions in the Lie ball can be represented in Dirichlet series by showing the equivalent fact that it can be constructed explicitly a discrete weakly sufficient set for the space of entire functions of exponential type on the complex light cone.
\end{abstract}

\section{Introduction.}

During the last two decades there has been developed extensively a concept of representing systems of exponents which shows, in particular, that every holomorphic function $f$ in a convex domain of $\mathbf{C}^{n}(n \geq 1)$ can be represented in the form of Dirichlet series

$$
f(z)=\sum_{k=1}^{\infty} c_{k} e^{\left\langle\lambda^{k}, z\right\rangle}
$$

where $\langle\cdot, \cdot\rangle$ is a scalar product.

The possibility of such a representation, as is well-known, relates to the existence of so-called sufficient and weakly sufficient sets (introduced and considered by Ehrenpreis [1], Taylor [9] and Schneider [8], among others). Roughly speaking, this relation can be formulated as follows: for certain spaces $H$ of holomorphic functions the representation (1.1) holds if and only if the set of frequencies $\left\{\lambda^{k}\right\}$ is weakly sufficient, or equivalently, as it turned out later, sufficient for the dual spaces $H^{*}$.

This means that in many cases it is important to have an explicit description of dual spaces. Then for solving the representation problem it suffices to deal with (weakly) sufficient sets. In this direction there were several papers in which the last sets have been considered in different spaces of holomorphic functions in convex domains of $\mathbf{C}^{n}$ (see, e.g., $[2,4]$ and references therein).

The first named author, wishing to have duality theorems (i.e., a description of

Received December 19, 1995

* The second named author was supported in part by the STEC grant of Sophia University and the CDE grant of the International Mathematical Union. 
dual spaces) for a non-convex set, has started to study analytic functionals (hyperfunctions) on the sphere. Since analytic functionals on the sphere can be regarded as continuous linear functionals on the space of complex harmonic functions in a neighbourhood of the Lie ball, a study of the last space is of great importance. On this subject he has obtained several results; in particular, in his recent joint work with $\mathrm{K}$. Fujita [6] there has been described the dual space of the space of complex harmonic functions in the Lie ball as the space of entire functions on the complex light cone.

The aim of this paper is to present an application of such results to the representation problem, namely to show that every harmonic functions in the Lie ball can be represented in Dirichlet series of the form (1.1), by constructing a weakly sufficient set for the space of entire functions on the complex light cone.

It should be noted that in the articles on sufficient sets mentioned above these sets are constructed for the spaces of either entire functions in $\mathbf{C}^{n}$ or holomorphic functions in convex domains of $\mathbf{C}^{n}$. The techniques used in those papers are essentially of convex domains, while the situation of the light cone considered in the present paper is of quite different nature.

ACKNOWLEDGMENTS. This paper was written during the second named author's stay in Sophia University, Tokyo, under its STEC grant. He wishes to thank the Department of Mathematics for the hospitality.

The authors are grateful to K. Fujita for her comments.

\section{Preliminaries on absolutely representing systems.}

A sequence $\left(x_{k}\right)$ of non-zero elements of a locally convex space $H$ is said to be an absolutely representing system in $H$ if any element $x$ from $H$ can be represented in the form of the series

$$
x=\sum_{1}^{\infty} c_{k} x_{k},
$$

which converges absolutely in the topology of $H$.

It should be noted that this concept is more general than concept of basis, where the uniqueness of representation is essentially required.

Studying absolutely representing systems Korobeinik has obtained remarkable results (see, e.g., $[2,3]$ ), in particular, criteria for a given sequence to be absolutely representing system in different functional spaces as well as significant properties of such systems. We refer the reader to these articles for having information in detail.

Below is one of the results from [3, Section 8, Corollary of Theorem F], which will be used in the present work.

Let $H_{k}$ be Banach spaces with the norms $\|\cdot\|_{m}$ such that $H_{m+1} \hookrightarrow H_{m}$ and $H=$ $\bigcap_{m=1}^{\infty} H_{m}$ is dense in each $H_{m}, m=1,2, \cdots$. Then $H=\lim$ proj $H_{m}$ is called a reduced 
projective limit of the Banach spaces $H_{m}$.

THEOREM A. Suppose that $H$ is the reduced projective limit of Banach spaces $H_{m}$ with the norms $\|\cdot\|_{m}, m=1,2, \cdots$. A system $X=\left\{x_{k}\right\}_{k=1}^{\infty}$ of non-zero elements is an absolutely representing system in $H$ if and only if $\forall m \geq 1 \exists p \geq 1, \exists C_{m} \in(0,+\infty)$ such that

$$
\sup _{x \in H}\left|y\left(\frac{x}{\|x\|_{p}}\right)\right| \leq C_{m} \sup _{k \geq 1}\left|y\left(\frac{x_{k}}{\left\|x_{k}\right\|_{m}}\right)\right|, \quad \forall y \in H^{*} .
$$

The inequality (2.1) can be interpreted as follows. Denote

$$
E_{m}=\left\{y \in H^{*} ;|y|_{m}=\sup _{x \in H}\left|y\left(\frac{x}{\|x\|_{m}}\right)\right|<+\infty\right\}, \quad m \geq 1,
$$

the Banach space with the norm $|\cdot|_{m}$. Then $H^{*}=\bigcup_{m=1}^{\infty} E_{m}$, and we can endow this space with the inductive topology:

$$
\left(H^{*}, \tau\right)=\lim \text { ind } E_{m}
$$

Denote further

$$
F_{m}=\left\{y \in H^{*} ;|\tilde{y}|_{m}=\sup _{k \geq 1}\left|y\left(\frac{x_{k}}{\left\|x_{k}\right\|_{m}}\right)\right|<+\infty\right\}, \quad m \geq 1,
$$

the seminormed space with the prenorm $|\tilde{y}|_{m}$.

Obviously, $E_{m} \subset F_{m} \subset H^{*}, \forall m \geq 1$, and this implies that $H^{*}=\bigcup_{m=1}^{\infty} F_{m}$. Consequently, the space $H^{*}$ can be endowed with the weaker inductive topology

$$
\left(H^{*}, \mu\right)=\lim \text { ind } F_{m} \text {. }
$$

It is easily seen that two topologies $\tau$ and $\mu$ are equivalent if and only if (2.1) holds. So we arrived to the fact that the following statements are equivalent for any system $X=\left\{x_{k}\right\}$ of non-zero elements of the reduced projective limit $H:$ i) $X$ is an absolutely representing system in $H$; ii) the relation (2.1) holds; iii) $\tau=\mu$.

\section{Spaces of complex harmonic functions in Lie balls and their dual.}

Recalling some notations we refer the readers to [5-7] for the general background of this section.

Let $\|x\|$ be the Euclidean norm on $\mathbf{R}^{n+1}$. The cross norm $L(z)$ on $\mathbf{C}^{n+1}$ corresponding to $\|x\|$ is the Lie norm defined by

$$
L(z)=L(x+i y)=\left\{\|x\|^{2}+\|y\|^{2}+2 \sqrt{\|x\|^{2}\|y\|^{2}-\langle x, y\rangle^{2}}\right\}^{1 / 2},
$$

where $z=x+i y, x, y \in \mathbf{R}^{n+1}$ and $\langle x, y\rangle=x_{1} y_{1}+\cdots+x_{n+1} y_{n+1}$. We denote by $L^{*}(z)$ the dual Lie norm: 


$$
L^{*}(z)=\sup _{L(\zeta) \leq 1}|\langle z, \zeta\rangle|=\frac{1}{\sqrt{2}}\left\{\|x\|^{2}+\|y\|^{2}+\sqrt{\left(\|x\|^{2}-\|y\|^{2}\right)^{2}+4\langle x, y\rangle^{2}}\right\}^{1 / 2} .
$$

Note that $\|z\| / \sqrt{2} \leq L^{*}(z) \leq L(z) \leq 2 L^{*}(z)$ for all $z \in \mathbf{C}^{n+1}$.

The open unit Lie ball is denoted by $\widetilde{B}$, i.e., $\widetilde{B}=\left\{z \in C^{n+1} ; L(z)<1\right\}$.

Let $\mathcal{O}(R \widetilde{B}), R>0$, be the space of holomorphic functions in $R \widetilde{B}$ with the topology of uniform convergence on compact subsets of $R \widetilde{B}$. As is well-known, it is a FréchetSchwartz space.

Consider the space of complex harmonic functions in $R \widetilde{B}$ :

$$
\mathcal{O}_{\Delta}(R \widetilde{B})=\left\{f \in \mathcal{O}(R \widetilde{B}) ; \Delta f=\left(\frac{\partial^{2}}{\partial z_{1}^{2}}+\frac{\partial^{2}}{\partial z_{2}^{2}}+\cdots+\frac{\partial^{2}}{\partial z_{n+1}^{2}}\right) f=0\right\} .
$$

Since this space is a closed subspace of the space $\mathcal{O}(R \widetilde{B})$, it also is a Fréchet-Schwartz space.

Furthermore, take and fix some sequence $0<\left(q_{m}\right) \uparrow 1$. Let

$$
H_{m}=\left\{f \in \mathcal{O}_{\Delta}\left(R q_{m} \widetilde{B}\right) \cap C\left(\overline{R q_{m} \widetilde{B}}\right)\right\}, \quad m \geq 1 .
$$

Then, due to the homogeneity of the continuous Laplace operator, we can easily verify that the space $\mathcal{O}_{\Delta}(R \widetilde{B})$ is the reduced projective limit of the Banach spaces $H_{m}$.

We denote

$$
\tilde{S}_{0}=\left\{\zeta \in \mathbf{C}^{n+1} ; \zeta^{2}=\zeta_{1}^{2}+\zeta_{2}^{2}+\cdots+\zeta_{n+1}^{2}=0\right\}
$$

the complex light cone.

The Cauchy kernel is defined as follows:

$$
K_{0}(\xi, \zeta)=\frac{1+2\langle\xi, \zeta\rangle}{(1-2\langle\xi, \zeta\rangle)^{n}}
$$

This kernel is a symmetric holomorphic function in the set

$$
\left\{(\xi, \zeta) \in \mathbf{C}^{n+1} \times \mathbf{C}^{n+1} ; L(\xi) L^{*}(\zeta)<1 / 2 \text { or } L^{*}(\xi) L(\zeta)<1 / 2\right\}
$$

Also note that since

$$
L(\zeta)=2 L^{*}(\zeta), \quad \forall \zeta \in \tilde{S}_{0},
$$

if $\zeta \in \tilde{S}_{0}$ then $K_{0}(., \zeta)$ is a holomorphic function in the set $\left\{\xi \in \mathbf{C}^{n+1} ; L(\xi) L(\zeta)<1\right\}$ and satisfies the Laplace equation in this set.

For an entire function $F$ on the complex light cone $\tilde{S}_{0}$ there is a Cauchy integral formula [11]:

$$
F(\xi)=\int_{M} F(\rho \zeta) K_{0}(\xi, \zeta / \rho) d M(\zeta), \quad \forall \rho>0, \forall \xi \in \tilde{S}_{0}, L(\xi)<\rho,
$$


where $d M$ is the normalized invariant measure on $M=\left\{\zeta \in \tilde{S}_{0} ; L(\zeta)=1\right\}$.

We introduce the following space of entire functions of exponential type $R$ on $\tilde{S}_{0}$ :

$$
\operatorname{Exp}\left(\widetilde{S}_{0} ; R\right)=\lim \text { ind } \widetilde{E}_{m},
$$

where

$$
\tilde{E}_{m}=\left\{F \in \mathcal{O}\left(\tilde{S}_{0}\right) ;|F|_{m}=\sup _{\tilde{S}_{0}} \frac{|F(\zeta)|}{e^{R q_{m} L^{*}(\zeta)}}<+\infty\right\}, \quad m \geq 1,
$$

and $0<\left(q_{m}\right) \uparrow 1$.

The Fourier-Borel transform of an analytic functional $T \in \mathcal{O}_{\Delta}^{*}(R \widetilde{B})$ is defined by

$$
\mathscr{F}^{S} T(\zeta)=\left\langle T_{z}, e^{\langle z, \zeta\rangle}\right\rangle, \quad \zeta \in \tilde{S}_{0}
$$

In [10] R. Wada obtained the following duality result (see also [7]).

THEOREM B. The Fourier-Borel transformation establishes a topological isomorphism between the strong dual of $\mathcal{O}_{\Delta}(R \widetilde{B})$, the $\operatorname{space} \mathcal{O}_{\Delta}^{*}(R \widetilde{B})$, and the $\operatorname{space} \operatorname{Exp}\left(\widetilde{S}_{0} ; R\right)$.

This duality theorem allows us, as will be seen in the next section, to prove the possibility of representation of complex harmonic functions in $R \widetilde{B}$ by Dirichlet series.

\section{Weakly sufficient sets on the complex light cone.}

Let $\Lambda$ be any subset of $\tilde{S}_{0}$. Then this subset and the corresponding sequence of seminormed spaces

$$
E_{m}^{\Lambda}=\left\{F \in \operatorname{Exp}\left(\tilde{S}_{0} ; R\right) ;|F|_{m}^{\Lambda}=\sup _{\Lambda} \frac{|F(\zeta)|}{e^{R q_{m} L^{*}(\zeta)}}<+\infty\right\}, \quad m \geq 1,
$$

where $0<\left(q_{m}\right) \uparrow 1$, generate in $\operatorname{Exp}\left(\tilde{S}_{0} ; R\right)$ another weaker inductive topology. If these two topologies are equivalent, then the set $\Lambda$ is called a weakly sufficient set for the space $\operatorname{Exp}\left(\widetilde{S}_{0} ; R\right)$. So the complex light cone $\tilde{S}_{0}$ itself is a weakly sufficient set for $\operatorname{Exp}\left(\tilde{S}_{0} ; R\right)$.

Note that

$$
\sup _{z \in R q_{m} \tilde{B}}\left|e^{\langle\zeta, z\rangle}\right|=e^{R q_{m} L^{*}(\zeta)}, \quad \forall \zeta \in \tilde{S}_{0}, \forall m \geq 1 .
$$

Applying Theorem A and the remark that follows to the spaces in Theorem B we obtain the following result.

Proposition 4.1. Let $\lambda^{k} \in \tilde{S}_{0}(k=1,2, \cdots)$. Then the system $\left(e^{\left\langle\lambda^{k}, z\right\rangle}\right)_{k=1}^{\infty}$ is absolutely representing in the space $\mathcal{O}_{\Delta}(R \widetilde{B})$ if and only if the set $\Lambda=\left\{\lambda^{k}\right\}_{k=1}^{\infty}$ is weakly sufficient for the space $\operatorname{Exp}\left(\tilde{S}_{0} ; R\right)$, i.e.,

$$
\forall m \in \mathbf{N} \exists s=s(m) \in \mathbf{N}, \quad \exists C=C(m)>0
$$




$$
\sup _{\zeta \in \tilde{S}_{0}} \frac{|F(\zeta)|}{e^{R q_{s} L^{*}(\zeta)}} \leq C \sup _{k \geq 1} \frac{\left|F\left(\lambda^{k}\right)\right|}{e^{R q_{m} L^{*}\left(\lambda^{k}\right)}}, \quad \forall F \in \operatorname{Exp}\left(\tilde{S}_{0} ; R\right) .
$$

The important question now is whether there exists such a sequence $\Lambda$. We proceed to study this problem.

In the paper [4], following Korobeinik's scheme in [2] for constructing discrete weakly sufficient sets in the space of entire functions of exponential type of one complex variable, the second named author has proved the explicit existence of the discrete set $\Lambda \subset \mathbf{C}^{n}$ that is weakly sufficient for the weighted space of entire functions in $\mathbf{C}^{n}$ and obtained theorems of representation of holomorphic functions in Dirichlet series.

As was already noted in the introduction, the situation considered in the present paper is quite different from those mentioned above: the complex light cone is never a domain in $\mathbf{C}^{n+1}$. Nevertheless, it turns out that the ideas for the domain case can be applied to the space $\operatorname{Exp}\left(\tilde{S}_{0} ; R\right)$ for construction of weakly sufficient sets.

For $F \in \operatorname{Exp}\left(\tilde{S}_{0} ; R\right)$ and $t>0$ we set

$$
\begin{gathered}
C_{t}=\left\{\zeta \in \tilde{S}_{0} ; L^{*}(\zeta)=t\right\}, \\
M_{t}(F)=\sup _{\zeta \in C_{t}}|F(\zeta)| .
\end{gathered}
$$

LeMma 4.2. Let $F \in \mathcal{O}\left(\tilde{S}_{0}\right)$. Then $M_{t}(F) \leq M_{s}(F)$ for $0<t \leq s$.

Proof. Take and fix $z \in C_{t}$. The expansion of $F(z)$ in a series of homogeneous polynomials shows that the function $F(\lambda z), \lambda \in \mathbf{C}$, is an entire function with respect to $\lambda$. Therefore, since $0<t<s$ we have

$$
|F(z)| \leq \sup _{|\lambda|=s / t}|F(\lambda z)| \leq M_{s}(F),
$$

and the desired inequality follows.

LEMMA 4.3. Let $F$ be an entire function on $\tilde{S}_{0}$ of exponential type $r_{0}$, i.e., for some $A \geqq 0$

$$
|F(\zeta)| \leq A \exp \left\{r_{0} L^{*}(\zeta)\right\}, \quad \forall \zeta \in \tilde{S}_{0}
$$

Then $\forall r>r_{0}$

$$
\underset{t \rightarrow \infty}{\liminf } \frac{M_{t+1}(F)}{M_{t}(F)} \leq e^{r}
$$

Proof. Since $F$ has the exponential type $r_{0}$ from the definition of $M_{t}(F)$ it follows that

$$
\log M_{t}(F)=\log \sup _{\zeta \in C_{t}}|F(\zeta)| \leq \log A+r_{0} t
$$


Hence,

$$
\liminf _{t \rightarrow \infty} \frac{\log M_{t}(F)}{t} \leq r_{0}
$$

Assume that (4.1) is not true. Then there is $r>r_{0}$ such that for all $t$ large enough - we have

$$
M_{t+1}(F) \geq e^{r} M_{t}(F)
$$

which implies that

$$
\liminf _{t \rightarrow \infty} \frac{\log M_{t}(F)}{t} \geq r>r_{0}
$$

This contradicts (4.2).

The following result plays a crucial role in our discussions.

Proposition 4.4. For any numbers $0<\rho_{1}<\rho$, if $z, w \in C_{\rho_{1}}$ and $F \in \mathcal{O}\left(\tilde{S}_{0}\right)$, then

$$
|F(z)-F(w)| \leq \frac{2 \sqrt{2} n(n+1)}{\rho\left(1-\rho_{1} / \rho\right)^{n+1}} M_{\rho}(F)|z-w| .
$$

Proof. Take $z, w \in \tilde{S}_{0}$ with $L(z)=L(w)=\rho_{1}<\rho$. Then, by the Cauchy intergal formula (3.2) on $\tilde{S}_{0}$ and Lemma 4.2 , we have

$$
\begin{aligned}
|F(z)-F(w)| & \leq \sup _{\eta \in \tilde{S}_{0}, L(\eta)=\rho}|F(\eta)| \cdot \sup _{\eta \in \tilde{S}_{0}, L(\eta)=\rho}\left|K_{0}\left(z, \bar{\eta} / \rho^{2}\right)-K_{0}\left(w, \bar{\eta} / \rho^{2}\right)\right| \\
& \leq M_{\rho}(F) \cdot \sup _{\eta \in \tilde{S}_{0}, L(\eta)=\rho}\left|K_{0}\left(z, \bar{\eta} / \rho^{2}\right)-K_{0}\left(w, \bar{\eta} / \rho^{2}\right)\right| .
\end{aligned}
$$

Consider $K_{0}(\xi, \zeta)$ with $\zeta \in \tilde{S}_{0}, L(\zeta)=1 / \rho$. As is well-known, in this case $K_{0}(\xi, \zeta)$ is a holomorphic function with respect to $\xi$ in the Lie ball $\rho \widetilde{B}=\left\{\xi \in \mathbf{C}^{n+1} ; L(\xi)<\rho\right\}$. Then the function

$$
f_{\zeta}(t)=K_{0}(t z+(1-t) w, \zeta), \quad t \in[0,1],
$$

is well defined, because $L(t z+(1-t) w) \leq t L(z)+(1-t) L(w)=\rho_{1}<\rho, \forall t \in[0,1]$.

Consequently, for $\xi=\bar{\eta} / \rho^{2}$ we have

$$
\left|K_{0}\left(z, \bar{\eta} / \rho^{2}\right)-K_{0}\left(w, \bar{\eta} / \rho^{2}\right)\right|=\left|f_{\zeta}(1)-f_{\zeta}(0)\right| \leq \sup _{t \in[0,1]}\left|f_{\zeta}^{\prime}(t)\right|
$$

Furthermore, on the one hand

$$
f_{\zeta}^{\prime}(t)=\sum_{j=1}^{n+1} \frac{\partial K_{0}}{\partial \xi_{j}} \cdot \frac{\partial \xi_{j}}{\partial t}=\sum_{j=1}^{n+1} \frac{\partial K_{0}}{\partial \xi_{j}} \cdot\left(z_{j}-w_{j}\right) .
$$

On the other hand, since $L(t z+(1-t) w) \leq \rho_{1}<\rho, \forall t \in[0,1]$, we have 


$$
\left|\frac{\partial K_{0}}{\partial \xi_{j}}\right| \leq \sup _{\xi \in \mathbf{C}^{n+1}, L(\xi) \leq \rho_{1}}\left|\frac{\partial K_{0}}{\partial \xi_{j}}\right|, \quad j=1,2, \cdots, n+1
$$

Hence,

$$
\left|f_{\zeta}^{\prime}(t)\right| \leq \sum_{j=1}^{n+1}\left\{\sup _{\xi \in \mathbf{C}^{n+1}, L(\xi) \leq \rho_{1}}\left|\frac{\partial K_{0}}{\partial \xi_{j}}\right|\right\}|z-w| .
$$

Now we estimate $\left|\frac{\partial K_{0}}{\partial \xi_{j}}\right|, j=1,2, \cdots, n+1$. By the definition (3.1),

$$
K_{0}(\xi, \zeta)=\frac{1+2\langle\xi, \zeta\rangle}{(1-2\langle\xi, \zeta\rangle)^{n}}, \quad \xi \in \mathbf{C}^{n+1}, L(\xi) \leq \rho_{1}<\rho, \zeta \in \tilde{S}_{0}, L(\zeta)=1 / \rho
$$

Then

$$
\frac{\partial K_{0}}{\partial \xi_{j}}=\frac{2 \zeta_{j}}{(1-2\langle\zeta, \zeta\rangle)^{n+1}}[(n+1)+2(n-1)\langle\xi, \zeta\rangle] .
$$

Since the Lie norm and its dual have a property that

$$
|\langle\xi, \zeta\rangle| \leq L(\xi) L^{*}(\zeta), \quad \forall \xi, \zeta \in \mathbf{C}^{n+1},
$$

and $L(\zeta)=2 L^{*}(\zeta), \forall \zeta \in \widetilde{S}_{0}$, we get

$$
2|\langle\xi, \zeta\rangle| \leq L(\xi) L(\zeta) \leq \frac{\rho_{1}}{\rho}<1 .
$$

Therefore, from (4.6) it follows that

$$
\begin{aligned}
\left|\frac{\partial K_{0}}{\partial \xi_{j}}\right| & \leq \frac{2\left|\zeta_{j}\right|}{\left(1-\rho_{1} / \rho\right)^{n+1}}\left[(n+1)+(n-1) \rho_{1} / \rho\right] \\
& \leq \frac{4 n|\zeta|}{\left(1-\rho_{1} / \rho\right)^{n+1}}=\frac{2 \sqrt{2} n}{\rho\left(1-\rho_{1} / \rho\right)^{n+1}} .
\end{aligned}
$$

From (4.5) and (4.7) it follows that

$$
\left|f_{\zeta}^{\prime}(t)\right| \leq \frac{2 \sqrt{2} n(n+1)}{\rho\left(1-\rho_{1} / \rho\right)^{n+1}}|z-w|, \quad \forall t \in[0,1], \forall \zeta \in \tilde{S}_{0}, L(\zeta)=1 / \rho .
$$

Combining (4.3)-(4.4) and (4.8) completes the proof of the proposition.

Now let $\left(t_{k}\right)_{k=1}^{\infty}$ be a sequence of positive numbers satisfying the following conditions

$$
\left(t_{k}\right) \uparrow+\infty ; \lim _{k \rightarrow \infty} \frac{t_{k+1}}{t_{k}}=1
$$

In the sequel in order to simplify computations we consider $\left(t_{k}\right)_{k=1}^{\infty}=(k)_{k=1}^{\infty}$ al- 
though the result is valid for a general case.

Take and fix some natural number $N_{0} \geq 1$ and mark on each $C_{K}\left(K=N_{0}, N_{0}+\right.$ $1, \cdots) l_{K}$ points $\zeta_{K, 1} ; \zeta_{K, 2} ; \cdots ; \zeta_{K, l_{K}}$ which form the $1 /(K+1)^{n+1}$-net of $C_{K}$.

We renumerate the obtained system of points $\left\{\zeta_{K, j} ; 1 \leq j \leq l_{K}, K \geq N_{0}\right\}$ under one sequence, denoted by $\Lambda=\left(\lambda^{k}\right)_{k=1}^{\infty}$, writing first all the points with $K=N_{0}$, and then with $K=N_{0}+1$, etc.

The meaning of a choice of the number $N_{0}$ is that the frequencies $\left(\lambda^{k}\right)$ can be chosen arbitrarily far away from the origin of coordinates.

Now we are able to state the main result of this paper.

THEOREM 4.5. The sequence $\Lambda=\left(\lambda^{k}\right)_{k=1}^{\infty}$ constructed above is a weakly sufficient set for all spaces $\operatorname{Exp}\left(\tilde{S}_{0} ; R\right), R>0$, or equivalently, the system $\left(e^{\left\langle\lambda^{k}, z\right\rangle}\right)_{k=1}^{\infty}$ is absolutely representing in all spaces $\mathcal{O}_{\Delta}(R \widetilde{B}), R>0$.

ProOF. Let $R$ be an arbitrary positive number. We recall that

$$
\operatorname{Exp}\left(\widetilde{S}_{0} ; R\right)=\lim \text { ind } \widetilde{E}_{m},
$$

where

$$
\tilde{E}_{m}=\left\{F \in \mathcal{O}\left(\tilde{S}_{0}\right) ;|F|_{m}=\sup _{\tilde{S}_{\mathrm{o}}} \frac{|F(\zeta)|}{e^{R q_{m} L^{*}(\zeta)}}<+\infty\right\}, \quad m \geq 1 .
$$

Consider $|F|_{m}$, which can be rewritten as follows

$$
|F|_{m}=\sup _{t>0} \frac{M_{t}(F)}{e^{t R q_{m}}}, \quad F \in \operatorname{Exp}\left(\tilde{S}_{0} ; R\right), m \geq N_{0}
$$

Let $K \geq N_{0}$ be a fixed natural number.

For $t \leq K$, due to Lemma 4.2, we have

$$
\frac{M_{t}(F)}{e^{t R q_{m}}} \leq \frac{M_{K}(F)}{e^{t R q_{m}}} \leq \frac{M_{K}(F)}{e^{K R q_{m}-1}} e^{K R}
$$

On the other hand, since

$$
\lim _{p \rightarrow \infty}\left\{(p+1) q_{m-1}-p q_{m}\right\}=-\infty,
$$

there is a positive $P_{m}$ satisfying

$$
P_{m} \geq \sup _{p \geq 1}\left\{(p+1) q_{m-1}-p q_{m}\right\} .
$$

Hence, for $t \in(p, p+1]$ we have

$$
\frac{M_{t}(F)}{e^{t R q_{m}}} \leq \frac{M_{p+1}(F)}{e^{t R q_{m}}} \leq \frac{M_{p+1}(F)}{e^{(p+1) R q_{m}-1}} e^{R\left[(p+1) q_{m-1}-p q_{m}\right]} \leq \frac{M_{p+1}(F)}{e^{(p+1) R q_{m}-1}} e^{R P_{m}} .
$$


Thus, if $C=\max \left\{e^{K R}, e^{R P_{m}}\right\}$, then for all $N \geq K$ we can write

$$
\begin{gathered}
X_{N}:=\sup _{0<t \leq N} \frac{M_{t}(F)}{e^{t R q_{m}}} \leq \max \left\{\sup _{0<t \leq K} \frac{M_{t}(F)}{e^{t R q_{m}}} ; \sup _{K \leq t \leq N} \frac{M_{t}(F)}{e^{t R q_{m}}}\right\} \\
\leq C \max \left\{\frac{M_{K}(F)}{e^{K R q_{m}-1}} ; \sup _{K \leq p \leq N} \frac{M_{p+1}(F)}{e^{(p+1) R q_{m}-1}}\right\} \\
=C \sup _{K \leq p \leq N+1} \frac{M_{p}(F)}{e^{p R q_{m}-1}}:=C Y_{N} .
\end{gathered}
$$

Furthermore, there exists $w_{p} \in C_{p}$ such that $\left|F\left(w_{p}\right)\right|=M_{p}(F)$. We can find some point $\zeta_{p, j_{0}}$ from $l_{p}$ points marked on $C_{p}$ that satisfies $\left|w_{p}-\zeta_{p, j_{0}}\right| \leq 1 /(p+1)^{n+1}$.

By Proposition 4.4, we have

$$
M_{p}(F)-\left|F\left(\zeta_{p, j_{0}}\right)\right|=\left|F\left(w_{p}\right)\right|-\left|F\left(\zeta_{p, j_{0}}\right)\right| \leq\left|F\left(w_{p}\right)-F\left(\zeta_{p, j_{0}}\right)\right| \leq \frac{A_{n}}{p+1} M_{p+1}(F),
$$

where $A_{n}=2 \sqrt{2} n(n+1)$.

Hence,

$$
\begin{gathered}
\frac{M_{p}(F)}{e^{p R q_{m}-1}}-\frac{\left|F\left(\zeta_{p, j_{0}}\right)\right|}{e^{p R q_{m}-1}} \leq A_{n} \frac{M_{p+1}(F)}{(p+1) e^{p R q_{m-1}}} \\
=\frac{M_{p+1}(F)}{e^{(p+1) R q_{m}-1}} \cdot \frac{A_{n} e^{R q_{m}-1}}{p+1} \leq \frac{M_{p+1}(F)}{e^{(p+1) R q_{m}-1}} \cdot \frac{A_{n} e^{R}}{p+1} .
\end{gathered}
$$

Consequently,

$$
\begin{gathered}
Y_{N}=\sup _{K \leq p \leq N+1} \frac{M_{p}(F)}{e^{p R q_{m}-1}} \\
\leq \sup _{K \leq p \leq N+1} \frac{\left|F\left(\zeta_{p, j_{0}}\right)\right|}{e^{p R q_{m}-1}}+\sup _{K \leq p \leq N+1}\left\{\frac{A_{n} e^{R}}{p+1} \cdot \frac{M_{p+1}(F)}{e^{(p+1) R q_{m}-1}}\right\} .
\end{gathered}
$$

Furthermore, since $\zeta_{p, j_{0}} \in C_{p}$ we have

$$
\frac{\left|F\left(\zeta_{p, j_{0}}\right)\right|}{e^{p R q_{m}-1}} \leq \sup _{\lambda^{k} \in C_{p}} \frac{\left|F\left(\lambda^{k}\right)\right|}{e^{p R q_{m}-1}} .
$$

Choose $K \geq N_{0}$ so that

$$
\frac{A_{n} e^{R}}{p+1} \leq \frac{1}{4}, \quad \forall p \geq K
$$

Then, denoting

$$
T_{N}=\sup _{K \leq p \leq N+1} \sup _{\lambda^{k} \in C_{p}} \frac{\left|F\left(\lambda^{k}\right)\right|}{e^{p R q_{m}-1}}
$$


we obtain

$$
Y_{N} \leq T_{N}+\frac{1}{4} Y_{N}+\frac{A_{n} e^{R}}{p+1} \cdot \frac{M_{N+2}(F)}{e^{(N+2) R q_{m-1}}},
$$

or equivalently,

$$
\frac{3}{4} Y_{N} \leq T_{N}+\frac{A_{n} e^{R}}{p+1} \cdot \frac{M_{N+2}(F)}{e^{(N+2) R q_{m}-1}}
$$

Now take and fix a number $\sigma>r_{0}$, where $r_{0}$ is the type of the function $F$, i.e.,

$$
|F(\zeta)| \leq A \exp \left\{r_{0} L^{*}(\zeta)\right\}, \quad \forall \zeta \in \tilde{S}_{0} .
$$

By Lemma 4.3, there exists $\left(p_{j}\right) \uparrow+\infty$ such that

$$
M_{p_{j}+1}(F) \leq e^{\sigma} M_{p_{j}}(F), \quad \forall j \geq 1 .
$$

In (4.10) putting $N=p_{j}, p_{j}+1, \cdots$, since for all $j$ large enough

$$
\frac{A_{n} e^{R+2 \sigma}}{p_{j}+1} \leq \frac{1}{4}
$$

we then have

$$
\begin{gathered}
\frac{3}{4} Y_{p_{j}} \leq T_{p_{j}}+\frac{A_{n} e^{R}}{p_{j}+1} \cdot \frac{M_{p_{j}+2}(F)}{e^{\left(p_{j}+2\right) R q_{m-1}}} \leq T_{p_{j}}+\frac{A_{n} e^{R+2 \sigma}}{p_{j}+1} \cdot \frac{M_{p_{j}}(F)}{e^{p_{j} R q_{m-1}}} \\
\leq T_{p_{j}}+\frac{1}{4} \frac{M_{p_{j}}(F)}{e^{p_{j} R q_{m}-1}} \leq T_{p_{j}}+\frac{1}{4} Y_{p_{j}},
\end{gathered}
$$

which is equivalent to

$$
Y_{p_{j}} \leq 2 T_{p_{j}}, \quad \text { for all } j \text { large enough . }
$$

Combining (4.9) and (4.11) we get

$$
X_{p_{j}} \leq 2 C T_{p_{j}} .
$$

Note that $|F|_{m}^{\Lambda}$ can be rewritten as follows

$$
|F|_{m}^{\Lambda}=\sup _{p \geq 1} \sup _{\lambda^{k} \in C_{p}} \frac{\left|F\left(\lambda^{k}\right)\right|}{e^{p R q_{m}}} .
$$

If $F \in \tilde{E}_{m}$, then in (4.12) letting $p_{j} \rightarrow \infty$ we get

$$
|F|_{m} \leq 2 C \sup _{p \geq K} \sup _{\lambda^{k} \in C_{p}} \frac{\left|F\left(\lambda^{k}\right)\right|}{e^{p R q_{m}-1}} \leq 2 C|F|_{m-1}^{\Lambda} .
$$

On the other hand, if $F \in \operatorname{Exp}\left(\tilde{S}_{0} ; R\right)$, but $F \notin \tilde{E}_{m}$, then 


$$
|F|_{m}=\lim _{j \rightarrow \infty} X_{p_{j}}=+\infty
$$

and from (4.12) it follows that $|\boldsymbol{F}|_{m-1}^{\Lambda}=+\infty$, too.

Thus, we always have

$$
|F|_{m} \leq 2 C|F|_{m-1}^{\Lambda}, \quad \forall F \in \operatorname{Exp}\left(\tilde{S}_{0} ; R\right),
$$

which, due to Proposition 4.1, completes the proof of the theorem.

\section{References}

[1] L. EhrenPreis, Analytically uniform spaces and some applications, Trans. Amer. Math. Soc. 101 (1961), 52-74.

[2] Yu. F. Korobełnik, Representing systems, Izv. Akad. Nauk SSSR Ser. Mat. 42 (1978), 325-355. English transl.: Math. USSR-Izv. 12 (1978), 309-335.

[3] Yu. F. KoROBEINIK, Inductive and projective topologies. Sufficient sets and representing systems (Russian), Ibid. 50 (1986), 539-565. English transl.: Math. USSR-Izv. 28 (1987), 529-554.

[4] Lê Hai Khôi, Espaces conjugués, ensembles faiblement suffisants discrets et systèmes de représentation exponentielle, Bull. Sci. Math (2), 113 (1989), 309-347.

[5] M. Mовiмото, Analytic functionals on the sphere and their Fourier-Borel transformations, Banach Center Publ. 11 (1983), 223-250.

[6] M. Morimoto and K. Funta, Analytic functionals and entire functionals on the complex light cone, Hiroshima Math. J. 25 (1995), 493-512.

[7] M. MoRimoto and K. Funita, Conical Fourier-Borel transformations for harmonic functionals on the Lie ball, Banach Center Publ. (to appear).

[ 8 ] D. M. SCHNEIDER, Sufficient sets for some spaces of entire functions, Trans. Amer. Math. Soc. 197 (1974), 161-180.

[9] B. A. TAYLOR, Discrete sufficient sets for some spaces of entire functions, Trans. Amer. Math. Soc. 163 (1972), 207-214.

[10] R. WADA, On the Fourier-Borel transformations of analytic functionals on the complex sphere, Tôhoku Math. J. 38 (1986), 417-432.

[11] R. WADA and M. Morimoto, A uniqueness set for the differential operator $\Delta_{z}+\lambda^{2}$, Tokyo J. Math. 10 (1987), 93-105.

\section{Present Addresses:}

Mitsuo MORIMOTO

Department of Mathematics, Sophia University,

KiOICHO, ChIYODA-KU, TOKYO, 102 JAPAN.

e-mail: morimoto@mm.sophia.ac.jp

LÊ HAI KHôI

HANOI INSTITUTE OF INFORMATION TECHNOLOGY, Nghia Do, Tu Liem, HaNoI 10000, Vietnam.

e-mail: lhkhoi@ioit.ncst.ac.vn 